\title{
The first cut is not the deepest
}

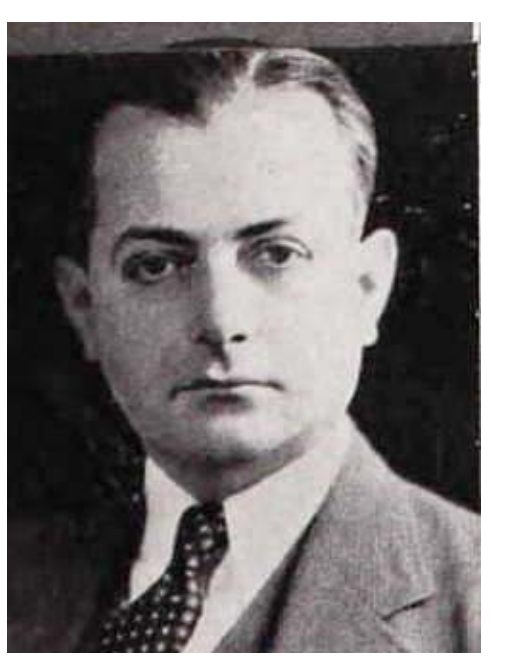

Earl Calvin Padgett

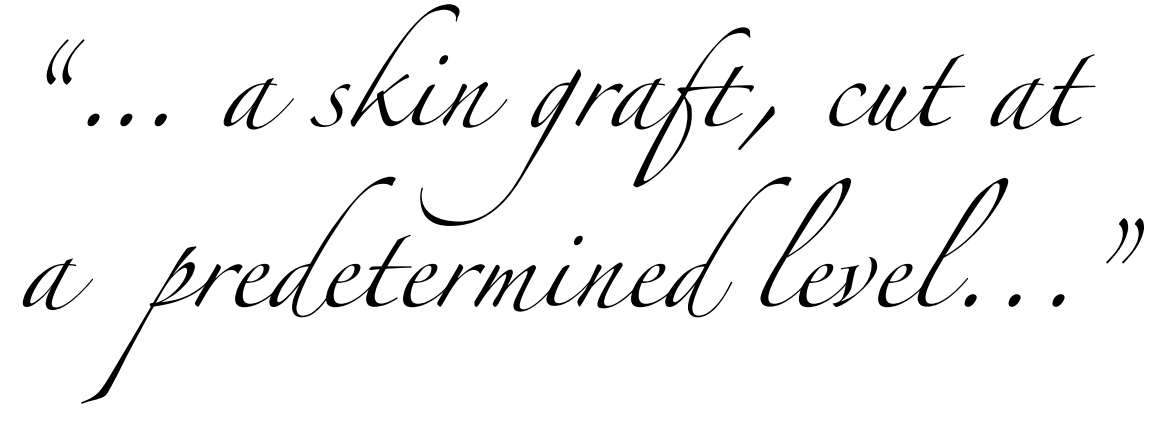

Background

1938. Calibrated dermatome of Padgett \& Hood. 1939. Asserts consistency and accuracy in depth of split-thickness skin graft (STSG) harvested.

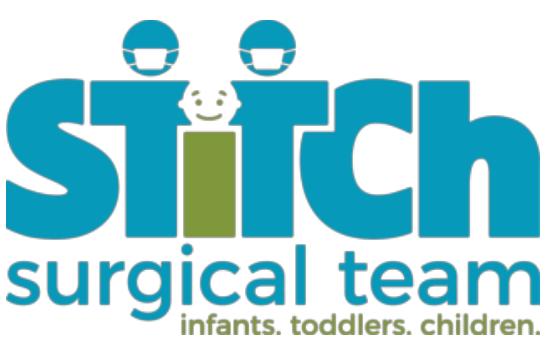

Dr Craig A McBride Margit Kempf Prof. Roy Kimble Dr Kellie Stockton

Centre For Children's Burns \& Trauma Research

\section{Objective}

Prospective cohort of STSC from children, to determine if the assertion is indeed true, or whether there are any predictable determinants of a thicker or thinner STSC

\section{Method}

101 patients

168 STSC

4 experienced surgeons

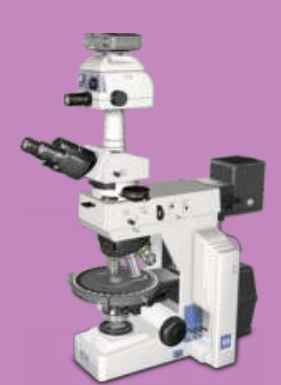

central biopsy blocked and cut, digitally measured

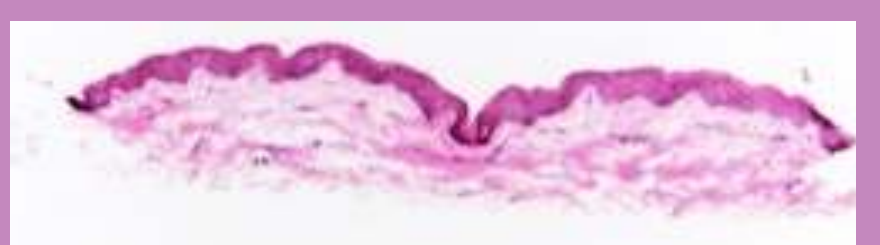

140 STSG @ 0.007" statistical analysis; ?accuracy ?predictors

\section{Results}

Comparison of STSG Thickness by Surgeon, and Combined Data (all surgeons)
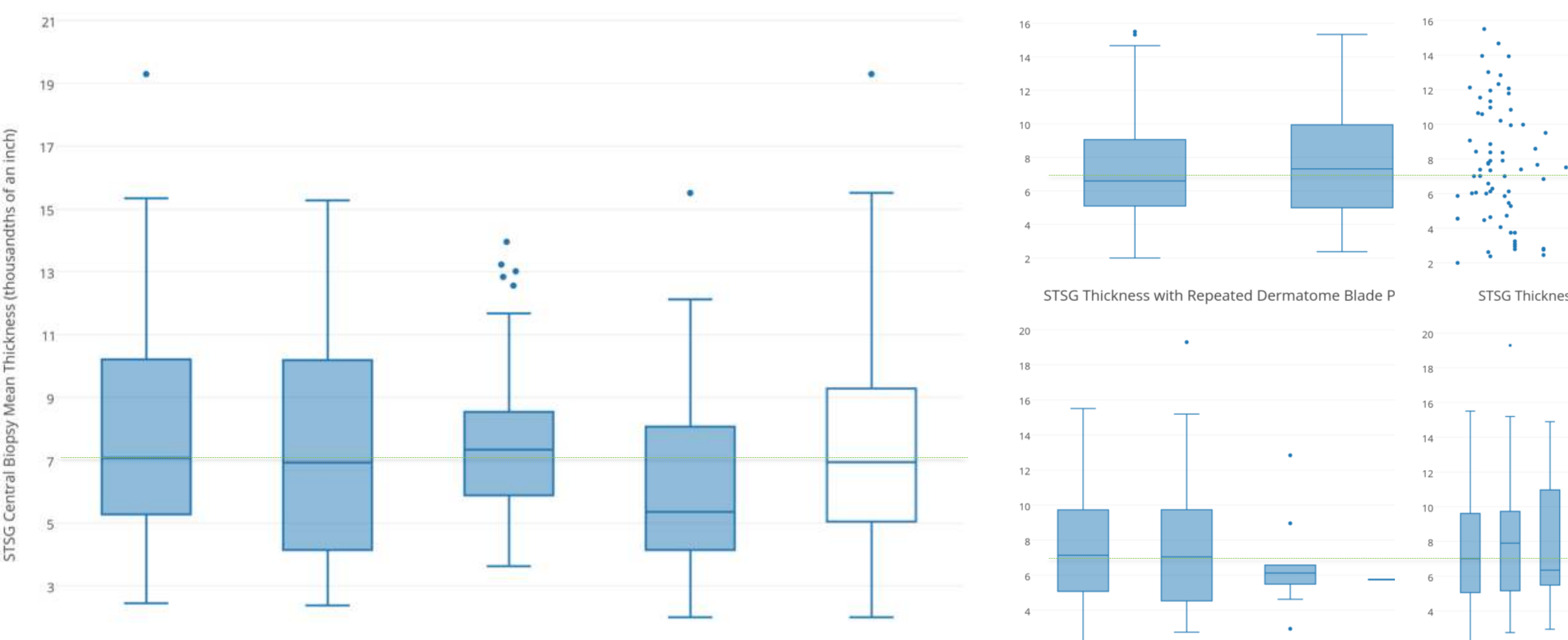

\section{Key Findings}

Median thickness accurate to dialed thickness at 0.007"
Broad range of STSC. $50 \%$ of STSC in the range 0.005 " - 0.009"
No pre-operative or intraoperative predictors of STSC thickness
Padgett was partly correct regarding accuracy
Possible implications in dressings trials using donor site wounds 